\title{
Predictability analysis of $\alpha$ decay formulae and the $\alpha$ partial half-lives of exotic nuclei
}

\author{
Nabanita Dasgupta-Schubert ${ }^{1, *}$ \\ ${ }^{1}$ Laboratorio de Biofísicoquímica y Estudios de Radiación, Facultad de Ciencias Físico Matemáticas, \\ Universidad Michoacana de San Nicolás de Hidalgo, Cd. Universitaria, \\ Morelia, Michoacán 58060, Mexico
}

\begin{abstract}
We examine the ability of 3 phenomenological alpha decay formulae, the Generalised Liquid Drop Model, the SobiczewskiParkhomenko and the Viola-Seaborg-Sobiczewski formulae, to predict the $\alpha$ partial half-lives $\mathrm{T}_{\alpha}$ of 100 exotic alpha radioactive nuclei by the statistical quantification of their accuracy and precision. These quantities were derived using a method based on standard experimental quality assurance wherein the alpha spectroscopic data of 313 well-established alpha decaying nuclei (calibration and test data sets) were used. Experimental masses as well as Finite Range Droplet Model masses were used to compute $Q_{\alpha}$. Improved coefficients for the three formulae were derived resulting in modified formulae. A simple linear optimization allowed adjustment of the modified formulae for the insufficient statistics of the odd-even and odd-odd decays of the calibration data set, without changing the modified formulae. Relatively better figures of merit for the odd-odd and the SHE were obtained using the modified GLDM formula.
\end{abstract}

\section{Introduction}

Alpha decay is the dominant decay mode and often the only one, of Super Heavy Elements (SHE) and highly neutron deficient medium mass nuclei, collectively termed 'exotic nuclei'. The SHEs are synthesized by heavy-ion fusion reactions at high power accelerators at "one-atom-at-a-time" levels with beams ranging from oxygen to zinc [1-3]. The SHEs decay by a succession of alphas that terminate in the spontaneous fission (SF) of the ultimate decay product closest to the line of stability. If this last product is a wellstudied nucleus, the identity of the progenitors is secured by the number of $\alpha$ decays in the genetic chain (each decay subtracts the proton number $\mathrm{Z}$ and neutron number $\mathrm{N}$ by 2 each). Unique identification becomes more difficult if the decays end in SF in a previously uncharted region. It is for this reason that the theoretical predictions of the $\alpha$ decay spectroscopic parameters - the $\alpha$ half-life $\left(\mathrm{T}_{\alpha}\right)$ and decay energy $\left(\mathrm{E}_{\alpha}\right)$ - assume direct significance to experiment, viz. in their ability to corroborate the experimental

*Email: nita@ifm.umich.mx,$\underline{\text { nitaschubert@gmail.com }}$ 
determinations of the succession of $\alpha$ decays of the SHE chain and thereby aid identification.

Theoretically, the $\alpha$ particle is imagined to tunnel through the potential energy (PE) barrier leading from the parent nucleus's energy to the total energy of the daughter nucleus and $\alpha$ particle. Calculation of barrier penetrability leads to the $\alpha$ decay constant $\lambda_{\alpha}$ and therefrom $\mathrm{T}_{\alpha} \quad\left(\mathrm{T}_{\alpha}=\ln 2 / \lambda_{\alpha}\right)$. Several microscopic model calculations as well as phenomenological model derived analytical formulae have been developed and have been applied to the prediction of exotic nuclear $\alpha$ decays [4]. While the analytical formulae cannot supplant the microscopic models with their deep mechanistic analysis, they find practical utility. They allow rapid prediction of $\mathrm{T}_{\alpha}$ for the observation of systematics over a wide $\mathrm{Z}$ and mass number A region; facile upgrade of their coefficients with the expansion/improvement of experimental data; easy incorporation into on-line data acquisition and analysis softwares requiring fewer cpu/memory resources. Three wellknown analytical formulae are G. Royer's (R) formula based on the Generalized Liquid Drop Model (GLDM) [5], the Viola-Seaborg-Sobiczewski (VSS) formula [6,7] and the Sobiczewski-Parkhomenko (SP) formula [8]. The latter two have been used in the experimental design of SHE synthesis: e.g. the VSS formula by the Dubna group [9], the SP formula by the Darmstadt-Dubna group [10].

The analytical formula typically relates $\log \left(\mathrm{T}_{\alpha}\right)$ with the $\mathrm{Q}_{\alpha}$ and the $(\mathrm{Z}, \mathrm{A})$ of the parent nuclide, with the coefficients of the terms being obtained from fits to the logs of the experimentally known half-lives, $\mathrm{T}_{\alpha}{ }^{\mathrm{exp}}$, of a set of nuclei that constitutes the 'primary basis set'. The smallness of the deviation between the calculated half-life $\left(\mathrm{T}_{\alpha}{ }^{\text {calc }}\right)$ and the $\mathrm{T}_{\alpha}{ }^{\exp }$ of the target nuclide set, e.g. a set of exotic $\alpha$ emitters, is taken to be a measure of the goodness of the formula towards the prediction of unknown half-lives (cf. [4] and [11] for details). This technique however is not a sufficiently sound method for testing the predictive accuracy of a given formula. Firstly, the target nuclide set being generally newly discovered exotics, possesses too few members that have well-characterized and corroborated $\alpha$ spectroscopic and nuclear mass data, to warrant a reliable statistical evaluation of the formula. Secondly, the primary data set is generally chosen to include a large number of known $\alpha$ emitters that span a wide $(\mathrm{Z}, \mathrm{A})$ range. While the large number is statistically beneficial, it precludes the selection of an independent set with wellcharacterized data to test the accuracy of the formula (since this set is already contained in the primary basis set). In some cases, the primary basis set is expanded to include members of the target set, so that the goodness of the prediction of the half-lives of the target set is inherently compromised by this mutual non-exclusiveness, that could produce a misleadingly low deviation ( $c f$. [4] and [11] for details). Thirdly, the wideness of the Z,A range of the primary basis set, especially at its extrema, could lead to the inclusion of $\alpha$ emitters with poorly known spectroscopic and/or mass data (e.g. $\mathrm{Q}_{\alpha}$ surmised from systematics only) so that the coefficients so derived would carry over these latent uncertainties ( $c f$. [4] and [11] for details). Their effect on the $\mathrm{T}_{\alpha}$ calc will be amplified because of the logarithmic relationship. The predictive accuracy of the formula should be an important concern in its choice for incorporation into the experimental design of SHE synthesis and of radiochemical characterization of the element's properties, quite apart from its intrinsic importance as a means of valorising the formula.

Considering the analytical formula to be a part of the suite of methods of experimental research, we devise a protocol to determine its predictive accuracy basing our ansatz on the well-known IUPAC (International Union of Pure and Applied Chemists) guidelines of method validation in analytical chemistry [12]. In the following we present 
the analysis of the predictability of the R, the VSS, SP and their corresponding modified formulae with improved coefficients, a further linear optimisation of the modified formulae for primary data sets with low statistics, and the use of the modified formulae as well as their linearly optimized variant (where applicable) to obtain the $\mathrm{T}_{\alpha}$ of 100 exotic nuclei.

\section{Method}

\subsection{Formulae}

The R, VSS and SP formulae are given in the following. For all three formulae, the calculated $\alpha$-partial half-life $\mathrm{T}_{\alpha}$ is in seconds and the experimental $\alpha$-decay $\mathrm{Q}$ value, $\mathrm{Q}_{\alpha}$, is in $\mathrm{MeV}$.

12 parameter $\mathrm{R}$ formula:

$\log _{10}\left[\mathrm{~T}_{\alpha}(\mathrm{R})\right]=\mathrm{a}+\mathrm{b} \cdot \mathrm{A}^{1 / 6} \cdot \mathrm{Z}^{1 / 2}+\mathrm{c} \cdot \mathrm{Z} /\left(\mathrm{Q}_{\alpha}\right)^{1 / 2}$,

The values of the coefficients $a, b$ and $c$ for parent nuclides of $Z-N$ even-even (e-e), evenodd (e-o), odd-even (o-e) and odd-odd (o-o) respectively, were $(-25.31,-1.1629,1.5864)$; $(-26.65,-1.0859,1.5848)$; (-25.68, -1.1423, 1.592); and $(-29.48,-1.113,1.6971)$.

7 parameter VSS formula:

$\log _{10}\left[\mathrm{~T}_{\alpha}(\mathrm{VSS})\right]=(\mathrm{a} \cdot \mathrm{Z}+\mathrm{b}) \cdot\left(\mathrm{Q}_{\alpha}\right)^{-1 / 2}+(\mathrm{c} \cdot \mathrm{Z}+\mathrm{d})+\mathrm{h}_{\mathrm{log}}$,

where the coefficients $a, b$ and $c$ were obtained from fits to e-e $\alpha$-decaying nuclei. The parameter $\mathrm{h}_{\log }$ is the hindrance factor for nuclei with unpaired nucleons and is obtained by fits to odd nuclei.

Its value for e-e, e-o, o-e and o-o Z-N numbers of the parent nuclide were obtained, respectively, as $0,1.066,0.772,1.114$. The $a, b, c$ and $d$ parameters are, $a=1.66175 ; b$ $=-8.5166 ; \mathrm{c}=-0.20228 ; \mathrm{d}=-33.9069$.

5 parameter SP formula:

$\log _{10}\left[\mathrm{~T}_{\alpha}(\mathrm{SP})\right]=\mathrm{a} \cdot \mathrm{Z} \cdot\left(\mathrm{Q}_{\alpha}-\overline{\mathrm{E}}_{\mathrm{i}}\right)^{-1 / 2}+\mathrm{b} \cdot \mathrm{Z}+\mathrm{c}$,

The parameters, $a, b$ and $c$ are, respectively: $1.5372 ;-0.1607 ;-36.573$. The parameter $\bar{E}_{i}$ is given by,

$\overline{\mathrm{E}}_{\mathrm{i}}=0 \mathrm{MeV}(\mathrm{e}-\mathrm{e}) ; \overline{\mathrm{E}}_{\mathrm{i}}=\overline{\mathrm{E}}_{\mathrm{n}}=0.171 \mathrm{MeV}(\mathrm{e}-\mathrm{o}) ; \overline{\mathrm{E}}_{\mathrm{i}}=\overline{\mathrm{E}}_{\mathrm{p}}=0.113 \mathrm{MeV}(\mathrm{o}-\mathrm{e}) ; \quad \overline{\mathrm{E}}_{\mathrm{i}}=\overline{\mathrm{E}}_{\mathrm{n}}+\overline{\mathrm{E}}_{\mathrm{p}}$ (o-o)

The coefficients of the aforesaid formulae were arrived at by fits to a large (Z,A) set of $\alpha$ emitters but the variability between the sets both in number and identity of nuclides considered, precludes a direct inter-comparison of accuracy. Additionally, all the considerations mentioned in $\S 1$ apply.

\subsection{Modified formulae and tests of predictability: the REF and TEST nuclide sets}

To validate a method or instrument, first there should be a calibration with certified standards in order to determine the relevant numerical parameters that determine the operation of the method/instrument. In the present case the 'calibration standards' are a set of Reference $\alpha$ decaying nuclei (the REF set) with very well-characterised spectroscopic and mass data. These are the nuclides used as "Alpha Particle Energy and Intensity Standards" for $\alpha$-spectrometry (p. 14064 of [12]): 78 nuclides with $Z=62-100, N=84-155$ of which 31 (e-e), 19(e-o), 15(o-e) and 13(o-o) are even (e) or odd (o) in Z and N numbers respectively. This is the primary basis set and is used to derive the coefficients of the R, VSS and SP formulae resulting in the modified formulae Rm, VSm and SPm. The REF is not a large basis set with a wide Z,N span. Hence the resulting formulae (see below and the tables 1-3), are not expected to produce a close agreement between $T_{\alpha}{ }^{\text {calc }}$ and $T_{\alpha}{ }^{\exp }$ for nuclei far outside the range of the REF set, i.e. for the exotic nuclei. However, that is not 
the intention of this work. The intention is to produce formulae with highly reliable coefficients that can be used to test the predictability of the formulae using an independent and approximately similar data set. The secondary objective of testing and optimising the modified formulae for predicting the $\mathrm{T}_{\alpha}$ of the exotic $\alpha$ emitters is treated in $\S 3.4$.

The second step in a method validation process is to test the calibrated method/instrument on a set of Standard Reference Materials (SRMs) which are standardised materials that share overall characteristics (such as the form of the matrix and its composition) with the calibration materials. We translate this procedure to the present work by selecting a set of well-studied $\alpha$ emitters, 235 in number, of which there are 78, 66, 55 and 36 respectively of the e-e, e-o, o-e and o-o parities ( $c f$. [11] for the full listing and the criteria of selection). This set is called the TEST and it covers a Z,A range close to the REF set. The coefficient of variation (CV) is an index of precision of measured values [13]. The average root mean square CV (RMSCV) of $\mathrm{T}_{\alpha}{ }^{\exp }$ and $\mathrm{Q}_{\alpha}{ }^{\exp }$ of the REF set for all parities were $0.105 \pm 0.032$ and $(1.737 \pm 2.602) \times 10^{-3}$ respectively while for the TEST set they were $0.216 \pm 0.024$ and $(3.847 \pm 1.853) \times 10^{-3}$. These low values justify their use as standards for calibration and benchmarking. ( $c f$. Table 1 in [4]). However, it must be mentioned that for the individual $(\mathrm{Z}, \mathrm{N})$ parent nuclear sets of REF, the maximum RMSCV for both $\mathrm{T}_{\alpha}$ and $\mathrm{Q}_{\alpha}$ was for the o-o set. This higher degree of uncertainty would carry over to the coefficients of the formulae and the resulting predictabilities. For o-o alpha emitters we expect a lower predictive ability of the formulae.

In this work the predictability of the formula is defined in terms of the statistical figure of merit (FOM), the Relative Error (RE) which is the 'trueness' or index of accuracy. The RE between experimental and calculated $\log _{10} \mathrm{~T}_{\alpha}$ is calculated as,

$\mathrm{RE}=\left(\log _{10} \mathrm{~T}_{\alpha}{ }^{\text {exp }}-\log _{10} \mathrm{~T}_{\alpha}{ }^{\text {calc }}\right) / \log _{10} \mathrm{~T}_{\alpha}{ }^{\exp }$,

Additionally, following Sobiczewski and Parkhomenko [8], their FOM f derived from the average value $\delta$ of the discrepancy $\delta$ which they describe as,

$\delta=\frac{1}{\mathrm{~N}} \sum_{\mathrm{i}=1}^{\mathrm{N}}\left|\log _{10}\left(T_{\alpha i}^{c a l c} / T_{\alpha i}^{\text {exp }}\right)\right|$

$\mathrm{f}=10^{\delta}$

was calculated [4]. Values of RE and f close to zero and one respectively, indicate that the formulae have good predictability.

The standard Levenberg-Marquardt non-linear multivariate regression fitting algorithm [14] was used to derive the coefficients of the formulae and their errors using the experimental $\alpha$ half-lives and errors of the REF basis set. These resulted in the "calibrated" formulae whose coefficients are given in $\S 3.1$.

Finally, the modified formulae were applied to 100 exotic alpha emitters utilizing their experimentally determined masses to determine $\mathrm{Q}_{\alpha}$ as well as the Finite Range Droplet model (FRDM) [15] masses.

\section{Results and Discussion}

\subsection{The modified formulae}

$\mathbf{R m}$. This is the modified Royer, 12 parameter formula: The values of the coefficients $\mathrm{a}, \mathrm{b}$ and $\mathrm{c}$ for (e-e), (e-o), (o-e) and (o-o) parent nuclides respectively were obtained as, $(-22.25 \pm 0.25, \quad-1.19 \pm 0.01, \quad 1.52 \pm 0.00) ; \quad(-27.77 \pm 0.28, \quad-1.00 \pm 0.00, \quad 1.58 \pm 0.00)$; $(-29.26 \pm 0.49,-1.03 \pm 0.00,1.62 \pm 0.01)$; and $(-28.30 \pm 0.05,-1.36 \pm 0.02,1.83 \pm 0.01)$.

VSm1. This is the modified VSS formula derived is the same manner as the original authors, using the REF data set, resulting in a 7 parameter formula: The values of the 
coefficients a,b, c and $\mathrm{d}$ for all parent $(\mathrm{Z}, \mathrm{N})$ sets are respectively, $1.48 \pm 0.01,2.07 \pm 1.15$, $0.21 \pm 0.00$ and $-30.53 \pm 0.45$. The value of $h_{\log }$ for the (e-e), (e-o), (o-e) and (o-o) parent are, $0.0,1.20 \pm 0.02,0.67 \pm 0.03$ and $1.10 \pm 0.10$ respectively.

VSm2. This is the modified VSS formula where the coefficients a,b,c, and $d$ have been derived explicitly for each parity set and where $\mathrm{h}_{\log }$ has been merged into $\mathrm{d}$ for the odd $\mathrm{Z}$ or $\mathrm{N}$ sets, resulting in a 16 parameter formula: The values of the coefficients a,b,c and $\mathrm{d}$ for the (e-e), (e-o), (o-e) and (o-o) parent are respectively, $(1.48 \pm 0.01,2.07 \pm 1.15,-0.21 \pm 0.00$, $30.53 \pm 0.45)$; $(1.52 \pm 0.00,4.32 \pm 0.23,-0.16 \pm 0.00,-36.27 \pm 0.13)$; $(1.61 \pm 0.04,-0.87 \pm 3.53$, $0.20 \pm 0.02,-34.58 \pm 1.54)$; (3.09 $\pm 0.01,-108.54 \pm 1.64,-0.81 \pm 0.01,11.33 \pm 0.75)$.

SPm1. This is the modified SP formula derived is the same manner as the original authors, using the REF data set, resulting in a 5 parameter formula: The values of the coefficients a,b, c for all parent nuclear $(\mathrm{Z}, \mathrm{N})$ sets are respectively, $1.51 \pm 0.00,-0.22 \pm 0.00,-29.42 \pm 0.17$, while the value of $E_{i}$ for the e-e, e-o, o-e and o-o parent were $0.0,0.16 \pm 0.00,0.10 \pm 0.00$, $0.27 \pm 0.01$

SPm2. This is the modified SP formula where the coefficients, a, b, c for all parent nuclides and $E_{i}$ for the odd nucleon number parents, were explicitly derived resulting in a 15 parameter formula. The values of the coefficients a, b, c and $E_{i}$ for the e-e, e-o, o-e and o-o parent are respectively, $(1.51 \pm 0.00,-0.22 \pm 0.00,-29.42 \pm 0.17,0.00) ;(1.55 \pm 0.00,-0.18 \pm 0.00$, $-34.10 \pm 0.28,0.04 \pm 0.01)$; (1.59 $\pm 0.05,-0.19 \pm 0.01,-35.21 \pm 0.03,0.02 \pm 0.10)$; $(10.85 \pm 0.63$, $2.00 \pm 0.09,-33.95 \pm 0.05,-13.50 \pm 0.82)$.

\subsection{Validation of the modified formulae}

The aforesaid modified formulae (all with the suffix 'm') were applied to the 235 nuclides of the TEST set. The standard deviations $\mathrm{s}(\mathrm{RE})$ on the RE values were obtained by the propagation of the experimental errors and the errors in the formula coefficients over the RE expression (eq. (4)). The RMSRE along with its corresponding RMS value of the standard deviation, $\operatorname{RMS}[\mathrm{s}(\mathrm{RE})]$, is shown in table 1. The RMSRE $\pm \operatorname{RMS}[\mathrm{s}(\mathrm{RE})]$ yields a measure of the accuracy of the various formulae in their prediction of the $\alpha$ half-life and the uncertainty of the predictability associated with the dispersion caused by the experimental and formula precision errors. The results for each formula are summarised for the 4 parity sets by the average $\mathrm{RMSRE} \pm \mathrm{RMS}[\mathrm{s}(\mathrm{RE})]$. Between the $\mathrm{m} 1$ and $\mathrm{m} 2$ variants, the $\mathrm{m} 2$ formulae have the lower values of the FOM. This is as expected because of the fewer number of adjustable parameters in the $\mathrm{m} 1$ formulae. A complete discussion on the $\mathrm{m} 1$ and $\mathrm{m} 2$ formulae are to be found in [4]. Taking the average RMSRE and the average $\mathrm{f}$ given in Table 1 into consideration, it would appear that the Rm formula, shows relatively the "highest" degree of closeness to experimental values. It possesses however, 12 adjustable parameters.

For certain nuclides, such as the e-e set for all formulae and the e-o set for the $\mathrm{SPm} 1$, the RMSRE $\pm \mathrm{RMS}[\mathrm{s}(\mathrm{RE})]$ values are quite high. These high values are the result of large deviations near certain $\mathrm{N}$ numbers. All three phenomenological formulae essentially incorporate shell effects only implicitly via the $\mathrm{Q}_{\alpha}$ values. This work, unlike [8], does not exclude nuclei near neutron shell closures. Only in the case of the SPm1 and SPm2 for the e-o set, the nuclide ${ }^{215} \mathrm{Th}$, was dropped because of its rather large RE. Figure 1 shows the distribution of RE with respect to $\mathrm{N}$ for all nuclides in TEST and for all formulae. Marked non-zero magnitudes of the RE occur in regions of $\mathrm{N}$ near the shell closure numbers of 82 , 126 and $\mathrm{N}=146$ ("deformed magic") as well as around the regions of $\mathrm{N}=88-90$ ("shape transition"), $\mathrm{N}=96-98$ ("loss of collectivity and sub-shell gaps") and $\mathrm{N}=134$ ("static octupole deformation"). 
Table 1. The RMSRE with its standard deviation RMS[s(RE)] and the $\mathrm{f}$ values for the different modified formulae applied to the TEST nuclides.

\begin{tabular}{|c|c|c|c|c|c|}
\hline Formula & $\mathrm{Z}, \mathrm{N}$ & $\mathrm{RMSRE} \pm \mathrm{RMS}[\mathrm{s}(\mathrm{RE})]$ & Avg. RMSRE & $\mathrm{f}$ & Avg. $\mathrm{f}$ \\
\hline $\mathrm{Rm}$ & $\begin{array}{l}\text { e-e } \\
\text { e-o } \\
\text { o-e } \\
\text { o-o }\end{array}$ & $\begin{array}{l}3.812 \pm 2.084 \\
1.434 \pm 1.001 \\
0.693 \pm 0.484 \\
1.039 \pm 0.106\end{array}$ & $1.745 \pm 0.591$ & $\begin{array}{l}2.510 \\
4.100 \\
3.870 \\
5.873\end{array}$ & 4.088 \\
\hline VSm1 & $\begin{array}{l}\text { e-e } \\
\text { e-o } \\
\text { o-e } \\
\text { o-o }\end{array}$ & $\begin{array}{l}3.681 \pm 1.671 \\
2.593 \pm 2.071 \\
1.410 \pm 0.894 \\
1.302 \pm 0.594\end{array}$ & $2.247 \pm 0.717$ & $\begin{array}{l}2.358 \\
12.00 \\
6.160 \\
9.765\end{array}$ & 7.570 \\
\hline VSm2 & $\begin{array}{l}\text { e-e } \\
\text { e-o } \\
\text { o-e } \\
\text { o-o }\end{array}$ & $\begin{array}{c}(3.681 \pm 1.671) \\
1.036 \pm 0.545 \\
0.753 \pm 0.357 \\
1.081 \pm 0.321\end{array}$ & $1.638 \pm 0.456$ & $\begin{array}{c}(2.358) \\
3.328 \\
3.990 \\
8.082\end{array}$ & 4.440 \\
\hline SPm1 & $\begin{array}{l}\text { e-e } \\
\text { e-o } \\
\text { o-e } \\
\text { o-o }\end{array}$ & $\begin{array}{l}3.763 \pm 1.978 \\
1.675 \pm 1.260 \\
1.106 \pm 0.682 \\
1.257 \pm 0.546\end{array}$ & $1.950 \pm 0.626$ & $\begin{array}{l}2.448 \\
5.794 \\
4.570 \\
9.589\end{array}$ & 5.600 \\
\hline SPm2 & $\begin{array}{l}\text { e-e } \\
\text { e-o } \\
\text { o-e } \\
\text { o-o }\end{array}$ & $\begin{array}{c}(3.763 \pm 1.978) \\
1.106 \pm 0.905 \\
0.805 \pm 0.435 \\
1.426 \pm 0.167\end{array}$ & $1.775 \pm 0.556$ & $\begin{array}{c}(2.448) \\
3.588 \\
4.120 \\
27.25 \\
\end{array}$ & 9.352 \\
\hline
\end{tabular}

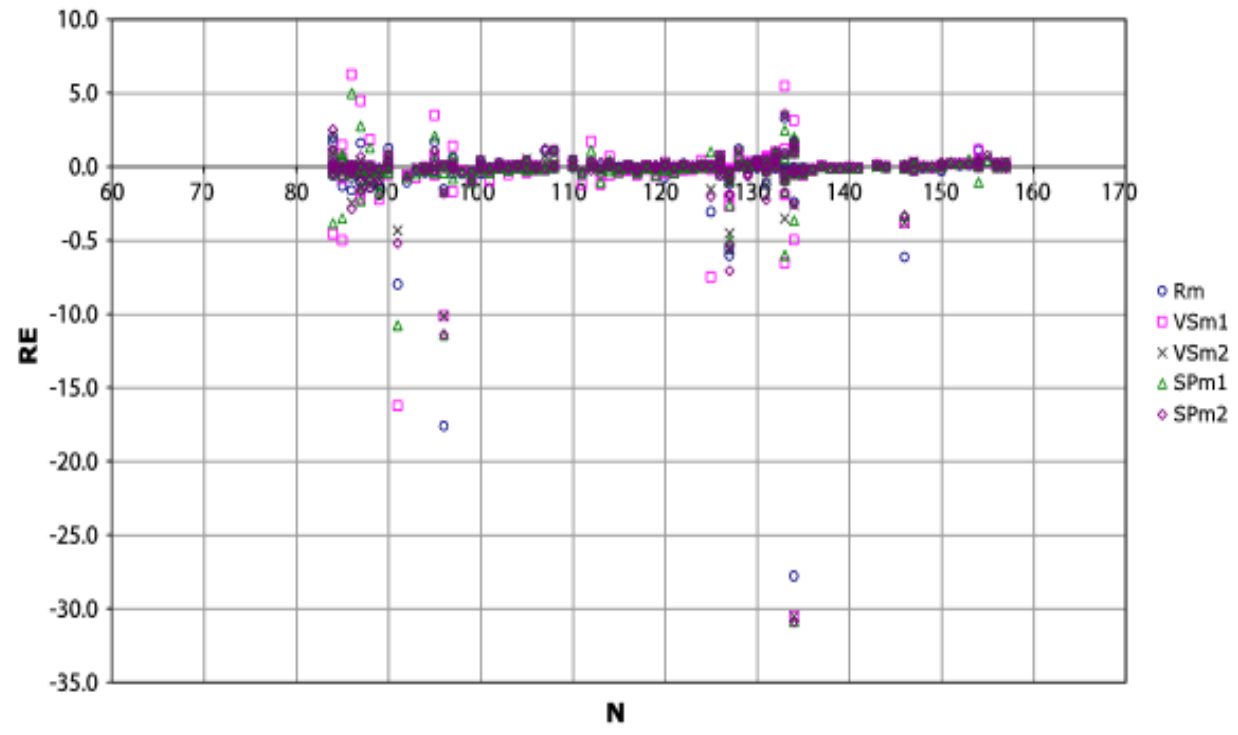

Figure 1. Plot of the relative error (RE) between the $\log _{10}$ of the experimental and calculated $\alpha$ decay half-lives of the TEST nuclei versus their neutron numbers, $\mathrm{N}$. 


\subsection{Linear optimization}

The REF nuclides or the 'calibration' set, suffer from not enough statistics for the odd parity sets, particularly for the o-o set, which affects the FOMs of the modified formulae. To artificially increase the statistics without deviating from the basic premise of using a fixed and highly reliable calibration basis set, we use the TEST set and linearly fit the $\log \left(\mathrm{T}_{\alpha}{ }^{\text {calc }}\right)$ derived from the aforesaid modified formulae to the $\log \left(\mathrm{T}_{\alpha}{ }^{\exp }\right)$ values for this set, to obtain the linearly regressed equation, $\log \left(\mathrm{T}_{\alpha}\right.$ lin-opt $)$. The latter represents the "statistically improved" version of the given modified formula.

$\log \left(\mathrm{T}_{\alpha}{ }^{\operatorname{lin}-\mathrm{opt}}\right)=\mu \cdot \log \left(\mathrm{T}_{\alpha}^{\text {calc }}\right)+\kappa$,

The values of $\mu$ and $\kappa$ for the linearly optimised Rm, VSm2 and SPm2 formulae are shown in Table 2 . The linearly optimised formulae can only be used for $\alpha$ emitters outside the range of REF and TEST.

Table 2. Values of the coefficients, $\mu$ and $\kappa$, of the linearly optimised modified formulae for the odd parity sets. The prefix 'Lin-opt' signifies the linearly optimised formula as per eqn. 7

\begin{tabular}{|c|c|c|c|}
\hline Formula & Z,N & $\mu$ & $\kappa$ \\
\hline \multirow{3}{*}{ Lin-opt-Rm } & e-o & 0.9913 & -0.2811 \\
& o-e & 1.0431 & 0.3491 \\
& o-o & 0.9093 & 0.5284 \\
\hline \multirow{3}{*}{ Lin-opt-VSm2 } & e-o & 0.9943 & -0.1805 \\
& o-e & 1.0555 & 0.3363 \\
& o-o & 0.8973 & 0.4120 \\
\hline \multirow{2}{*}{ Lin-opt-SPm2 } & e-o & 0.9988 & -0.2400 \\
& o-e & 1.0558 & 0.3631 \\
& o-o & 0.7489 & 1.1479 \\
\hline
\end{tabular}

\subsection{Application of the formulae: the EXOTIC nuclei}

We now compare the modified formulae along with their linearly optimised variants for the odd sets, for their ability to predict the $\mathrm{T}_{\alpha}$ of the exotic nuclei that span a $(\mathrm{Z}, \mathrm{A})$ range from $(68,156)$ to $(118,294)$. The complete listing is provided in [4]. It was seen that in general, the agreement between $\log \left(\mathrm{T}_{\alpha}{ }^{\text {calc }}\right)$ and $\log \left(\mathrm{T}_{\alpha}{ }^{\mathrm{exp}}\right)$ and between the calculated values themselves were the best for the nuclei in the middle of this range. This is reasonable given the fact that the coefficients of the formulae have been derived from the REF set, whose members largely belong to the middle of the aforesaid range. A secondary factor is that nuclides at the extrema of this range are generally the most recently discovered and/or the furthest from the line of stability, which implies that their spectroscopic data are relatively the less well-established. A third and important factor is the inadequacy of the formulae in treating shell-effects as demonstrated in $\$ 3.2$. Figure 2 shows the RE of the various formulae graphed against the $\mathrm{N}$ number of the exotic nucleus. The agreement is generally good but large deviations from zero arise in the $\mathrm{N}$ range of 145-175. Linear optimisation reduces the magnitudes of the deviations somewhat but their presence persists. These deviations could arise from the deformed shell gaps in this region. For example, Herzberg [16] points out the shell stabilization of SHE with $\mathrm{N}=152$ and $\mathrm{N}=162$.

Table 3 shows the RMSRE values for the modified and the linearly optimised formulae. Comparing between the modified formulae alone, one notices that the $\mathrm{Rm}$ formula has the best overall Avg. RMSRE, followed by the VSm2 and then by the SPm2. In fact the Rm for the Exotic nuclide set displays a lower Avg. RMSRE (1.209) than it did 
for the TEST set $(1.745 \pm 0.591)$ while the corresponding values for the VSm2 and SPm2 formulae are higher.

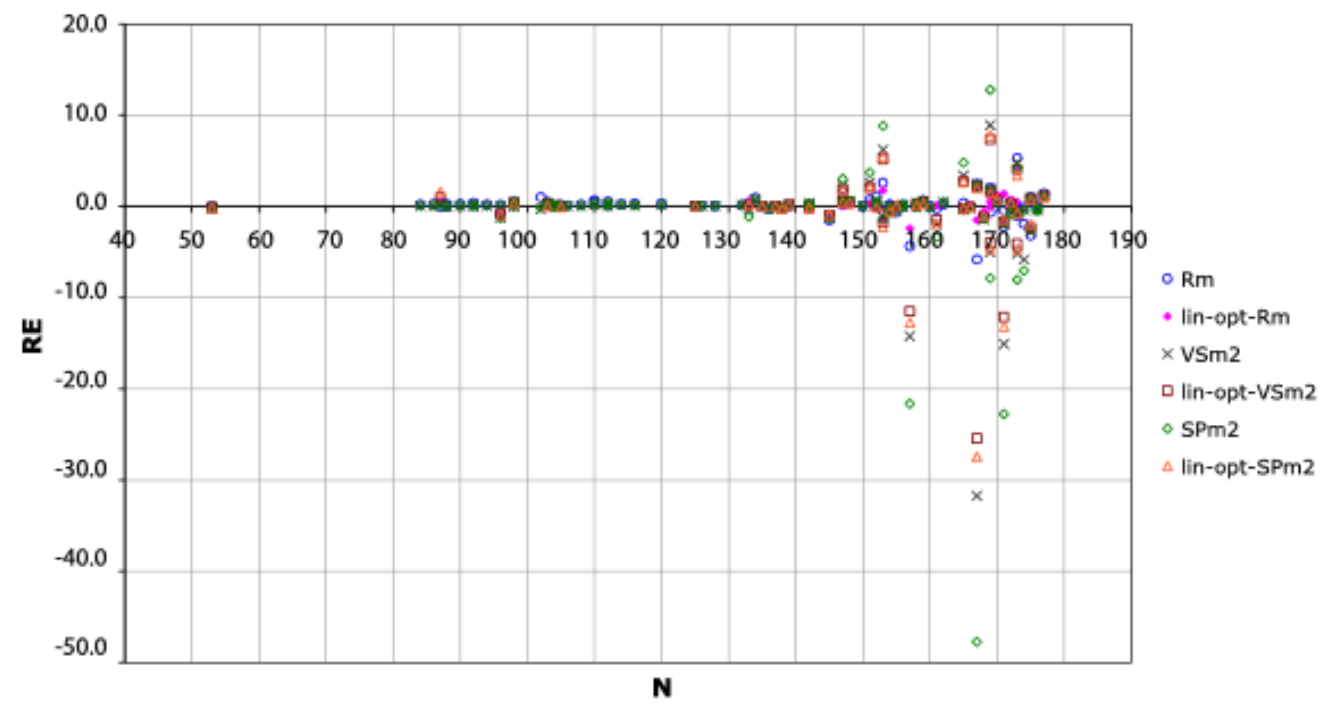

Figure 2. Variation of the RE between the $\log _{10}$ of the experimental and calculated $\alpha$ half-lives of the Exotic nuclei versus their neutron numbers, $\mathrm{N}$.

The $\mathrm{Rm}$ formula (eq. (1)) differs from the other two in containing the $\mathrm{A}^{1 / 6}$ term that arises from the GLDM formulation of the $\alpha$ decay where a quasi-molecular shape barrier is invoked [5]. The better fits to the SHE region underlines it greater applicability for heavy nuclides as against those in the TEST set that include lighter members.

The RMSRE for the odd parity sets are larger than for the e-e set attaining the highest values for the o-o set. This is consistent with the lower statistics for the odd nucleon sets of REF with the lowest being for the o-o set. The use of linear optimization improves the RMSRE for all odd nucleon sets and most markedly for the o-o set, resulting in a significant reduction of the Avg. RMSRE, for all 3 formulae. Taking the average of the RMSRE for the e-e set from the modified formula and the RMSRE of the odd nucleon sets from the linearly optimised formula, we obtain the Avg. RMSRE as 0.86 (Rm and Lin-optRm), 2.76 (VSm2 and Lin-opt-VSm2) and 2.92 (SPm2 and Lin-opt-SPm2). These are substantial reductions. Linear optimisation is therefore a useful technique to artificially "augment" the statistics of the calibration set (REF). Its advantage lies in the fact that the coefficients of the formula calculated using the reliable calibration data set, do not have to be recalculated by introducing new members into the calibration set. Rather the intact formula has only to be locally adjusted using simple linear regression. The larger the number of nuclei found with the degree of reliability of the TEST set near the region of the unknown nuclei, the better the linear optimisation is likely to be. 
Table 3. The RMSRE and its average value for the different modified formulae and their linearly optimised variants for the odd parity sets, as obtained for the 100 EXOTIC nuclides considered in this work. (N.B. The standard deviation RMS[s(RE)] could not be calculated because of insufficient information on the experimental errors)

\begin{tabular}{|c|c|c|c|c|}
\hline Formula & $\mathrm{Z,N}$ & RMSRE & $\begin{array}{l}\text { Avg. RMSRE (all } \\
\text { parities) }\end{array}$ & $\begin{array}{l}\text { Avg. RMSRE (odd } \\
\text { parities) }\end{array}$ \\
\hline $\mathrm{Rm}$ & $\begin{array}{l}\text { e-e } \\
\text { e-o } \\
\text { o-e } \\
\text { o-o }\end{array}$ & $\begin{array}{l}0.448 \\
1.841 \\
0.539 \\
2.006\end{array}$ & 1.209 & 1.462 \\
\hline Lin-opt-Rm & $\begin{array}{l}\text { e-o } \\
\text { o-e } \\
\text { o-o }\end{array}$ & $\begin{array}{l}1.518 \\
0.502 \\
0.961\end{array}$ & - & 0.994 \\
\hline VSm2 & $\begin{array}{l}\text { e-e } \\
\text { e-o } \\
\text { o-e } \\
\text { o-o }\end{array}$ & $\begin{array}{c}0.915 \\
1.674 \\
0.630 \\
10.099\end{array}$ & 3.330 & 4.134 \\
\hline Lin-opt-VSm2 & $\begin{array}{l}\text { e-o } \\
\text { o-e } \\
\text { o-o }\end{array}$ & $\begin{array}{l}1.485 \\
0.509 \\
8.141\end{array}$ & - & 3.378 \\
\hline SPm2 & $\begin{array}{l}\text { e-e } \\
\text { e-o } \\
\text { o-e } \\
\text { o-o }\end{array}$ & $\begin{array}{c}1.094 \\
1.547 \\
0.624 \\
15.187\end{array}$ & 4.613 & 5.786 \\
\hline Lin-opt-SPm2 & $\begin{array}{l}\text { e-o } \\
\text { o-e } \\
\text { o-o }\end{array}$ & $\begin{array}{l}1.331 \\
0.503 \\
8.816\end{array}$ & - & 3.550 \\
\hline
\end{tabular}

For completely unknown nuclei that potentially could be $\alpha$ emitters, no spectroscopic data would be available. To calculate the $\mathrm{T}_{\alpha}$, the $\mathrm{Q}_{\alpha}$ would have to be estimated from a suitable theoretical model of nuclear masses. In our previous work [11] we had used the Finite Range Droplet Model (FRDM95) masses of Möller et al [15]. The FRDM95 masses of the REF nuclides were used to obtain the $a, b$ and c coefficients of the Rm formula, designated here as Rm(FRDM). Their values for the e-e, e-o, o-e and o-o parent nuclides are respectively, $\{-7.2653,-1.2130,1.1441\} ;\{5.5997,-2.0451,1.3673\} ;\{-6.5247$, 1.5401. 1.3528\}: $\{-29.0028,-0.7217,1.4534\}$. It was found that using the FRDM95 derived $\mathrm{Q}_{\alpha} \mathrm{S}$ for the TEST nuclei in the Rm(FRDM) formula yielded better FOMs than using them in the Rm formula. In other words internal consistency is important. Recently Möller et al [17] have upgraded the FRDM calculations, that we designate here as FRDM12. Some SHE nuclides have also been newly synthesised and the spectroscopic data of others refined since the publication of [4] and [11]. Since the calculated $T_{\alpha}$ is very sensitive to the $Q_{\alpha}$ values, it would make sense to test the $\mathrm{Rm}(\mathrm{FRDM})$ using the $\mathrm{Q}_{\alpha}$ derived from FRDM95 and FRDM12 for these SHE nuclides to see how sensitive the procedure is to small changes in the mass estimates. The results are presented in Table 4. The experimental data were taken from the IAEA-NDS Table of Nuclides, https://wwwnds.iaea.org/relnsd/vcharthtml/VChartHTML.html. 
Except for the single e-e nuclide, ${ }^{294} \mathrm{Og}$, and for ${ }^{287} \mathrm{Mc}$, the FRDM12 produces the lower RMSRE despite marginal differences between the $\mathrm{Q}_{\alpha} \mathrm{S}$ obtained by the FRDM95 and FRDM12. The RMSRE values themselves, except for the o-o set, are comparable to those obtained with the Rm. The o-o set shows great disparities; the RMSRE values are much higher than those obtained from the Rm (Table 3), albeit the latter was on a different data set and a set that had much higher statistics. The deviation between the calculated and experimental $\mathrm{Q}_{\alpha}$ for the o-o set is not much greater than for the others. Hence here again, the greater errors in the Rm(FRDM) coefficients caused largely by the poor statistics of the $\mathrm{o}-\mathrm{o}$ REF set, is in evidence. Although a far bigger statistical data set is required before definitive conclusions can be arrived, the results suggest that (apart from the o-o nuclides) the FRDM12 mass calculation is reliable for the estimation of $\mathrm{T}_{\alpha}$ in uncharted regions.

Table 4. The $\log \left[\mathrm{T}_{\alpha}\right.$ calc $\left.(\mathrm{s})\right]$ calculated by the Rm(FRDM) for some recently synthesised SHE and SHE whose decay data have been upgraded. The $\mathrm{Q}_{\alpha} \mathrm{S}$ were calculated by the FRDM95 and FRDM12 masses ( $c f$. text).

\begin{tabular}{|c|c|c|c|c|c|c|c|}
\hline \multicolumn{8}{|c|}{ Even-even parent } \\
\hline Parent & $\mathrm{Q}_{\alpha}^{\exp }(\mathrm{MeV})$ & $\log \left[\mathrm{T}_{\alpha} \exp (\mathrm{s})\right]$ & $\begin{array}{c}\text { Theoretical } \\
\text { Mass }\end{array}$ & $\mathrm{Q}_{\alpha}{ }^{\text {calc }}(\mathrm{MeV}$ & $\begin{array}{c}\log \left[\mathrm{T}_{\alpha}{ }^{\text {calc }}\right. \\
(\mathrm{s})]\end{array}$ & $\mathrm{RE}$ & $\begin{array}{c}\text { RMSRE for } \\
\text { e-e parity } \\
\text { set }\end{array}$ \\
\hline \multirow[t]{2}{*}{${ }^{294}{ }_{118} \mathrm{Og}$} & \multirow[t]{2}{*}{$11.81 \pm 0.06$} & \multirow[t]{2}{*}{-2.74} & FRDM95 & 12.28 & -2.71 & 0.013 & 0.013 \\
\hline & & & FRDM12 & 12.37 & -2.85 & -0.038 & 0.038 \\
\hline \multicolumn{8}{|c|}{ Eve-odd parent } \\
\hline Parent & $\mathrm{Q}_{\alpha}^{\exp }(\mathrm{MeV})$ & $\left|\log \left[\mathrm{T}_{\alpha}{ }^{\exp }(\mathrm{s})\right]\right|$ & $\begin{array}{c}\text { Theoretical } \\
\text { Mass }\end{array}$ & $\mathrm{Q}_{\alpha}{ }^{\text {calc }}(\mathrm{MeV}$ & $\begin{array}{c}\log \left[\mathrm{T}_{\alpha}{ }^{\text {calc }}\right. \\
(\mathrm{s})]\end{array}$ & $\mathrm{RE}$ & $\begin{array}{l}\text { RMSRE for } \\
\text { e-o parity set }\end{array}$ \\
\hline \multirow[t]{2}{*}{$291_{116} \mathrm{LV}$} & \multirow[t]{2}{*}{$10.89 \pm 0.05$} & \multirow[t]{2}{*}{-2.20} & FRDM95 & 11.12 & -3.53 & -0.60 & 1.15 \\
\hline & & & FRDM12 & 11.02 & -3.31 & -0.51 & 0.94 \\
\hline \multirow[t]{2}{*}{${ }_{116}^{293} \mathrm{LV}$} & \multirow[t]{2}{*}{$10.68 \pm 0.05$} & \multirow[t]{2}{*}{-1.28} & FRDM95 & 10.94 & -3.20 & -1.51 & \\
\hline & & & FRDM12 & 10.78 & -2.85 & -1.32 & \\
\hline \multicolumn{8}{|c|}{ Odd-even parent } \\
\hline Parent & $\mathrm{Q}_{\alpha}^{\exp }(\mathrm{MeV})$ & $\log \left[\mathrm{T}_{\alpha}{ }^{\exp }(\mathrm{s})\right]$ & $\begin{array}{c}\text { Theoretical } \\
\text { Mass }\end{array}$ & $\mathrm{Q}_{\alpha}^{\text {calc }}(\mathrm{MeV}$ & $\begin{array}{c}\log \left[\mathrm{T}_{\alpha}^{\text {calc }}\right. \\
(\mathrm{s})]\end{array}$ & $\mathrm{RE}$ & $\begin{array}{l}\text { RMSRE for } \\
\text { o-e parity set }\end{array}$ \\
\hline \multirow[t]{2}{*}{${ }^{283}{ }_{113} \mathrm{Nh}$} & \multirow[t]{2}{*}{$10.48 \pm 0.11$} & \multirow[t]{2}{*}{-1.00} & FRDM95 & 9.36 & 1.51 & 2.51 & 1.49 \\
\hline & & & FRDM12 & 9.46 & 1.24 & 2.24 & 1.31 \\
\hline \multirow[t]{2}{*}{${ }^{289}{ }_{115} \mathrm{Mc}$} & \multirow[t]{2}{*}{$10.52 \pm 0.05$} & \multirow[t]{2}{*}{-0.66} & FRDM95 & 10.03 & 0.14 & 1.22 & \\
\hline & & & FRDM12 & 10.09 & -0.003 & 1.00 & \\
\hline \multirow[t]{2}{*}{${ }^{287}{ }_{115} \mathrm{Mc}$} & \multirow[t]{2}{*}{$10.74 \pm 0.06$} & \multirow[t]{2}{*}{-1.49} & FRDM95 & 10.25 & -0.34 & 0.77 & \\
\hline & & & FRDM12 & 10.22 & -0.27 & 0.82 & \\
\hline \multirow[t]{2}{*}{${ }^{293}{ }_{117} \mathrm{Ts}$} & \multirow[t]{2}{*}{$11.18 \pm 0.06$} & \multirow[t]{2}{*}{-1.85} & FRDM95 & 11.68 & -3.14 & -0.69 & \\
\hline & & & FRDM12 & 11.40 & -2.57 & -0.39 & \\
\hline
\end{tabular}




\begin{tabular}{|c|c|c|c|c|c|c|c|}
\hline \multicolumn{2}{|c|}{ Odd-odd parent } \\
\hline Parent & $\mathrm{Q}_{\alpha}{ }^{\exp }(\mathrm{MeV})$ & $\log \left[\mathrm{T}_{\alpha}{ }^{\exp }(\mathrm{s})\right]$ & $\begin{array}{l}\text { Theoretical } \\
\text { Mass }\end{array}$ & $\mathrm{Q}_{\alpha}{ }^{\text {calc }}(\mathrm{MeV})$ & $\begin{array}{c}\log \left[\mathrm{T}_{\alpha}{ }^{\text {calc }}\right. \\
(\mathrm{s})]\end{array}$ & $\mathrm{RE}$ & $\begin{array}{c}\text { RMSRE for } \\
\text { o-o parity } \\
\text { set }\end{array}$ \\
\hline${ }^{282}{ }_{113} \mathrm{Nh}$ & $10.78 \pm 0.05$ & -1.14 & FRDM95 & 9.78 & 3.88 & 4.41 & 11.38 \\
\cline { 5 - 8 } & & & FRDM12 & 10.02 & 3.25 & 3.86 & 10.70 \\
\hline${ }^{284}{ }_{113} \mathrm{Nh}$ & $10.23 \pm 0.11$ & -0.32 & FRDM95 & 9.15 & 5.64 & 18.68 & \\
\cline { 5 - 7 } & & & FRDM12 & 9.27 & 5.28 & 17.58 & \\
\hline${ }^{288}{ }_{115} \mathrm{Mc}$ & $10.63 \pm 0.05$ & -1.06 & FRDM95 & 10.13 & 3.64 & 4.43 & \\
\cline { 5 - 7 } & & & FRDM12 & 10.14 & 3.61 & 4.40 & \\
\hline
\end{tabular}

\section{Conclusion}

The predictability of 3 phenomenological formulae, the R, VSS and SP was quantitatively analysed in terms of statistical FOMs. The modified R formula with its linearly optimised variant was found to yield relatively better agreement with the experimental ground-state to ground-state $\alpha$ partial half-lives, particularly for the heavy $\alpha$ emitters. Substantially high deviations were found near neutron numbers that relate to shell closures/gaps. The use of the recently upgraded FRDM theoretically derived masses yielded generally acceptable agreement for some newly synthesised SHE and SHE whose decay data have been refined, except for o-o parents. The overall results suggest a need for improvement of the formulae to consider shell and angular momentum effects.

Since the time of Geiger and Nuttall [18] a large number of empirical or phenomenological alpha decay formulae have been proposed. Many purport to obtain good fits to the experimental alpha decay half-lives. However, till now, it has been difficult to perform a critical inter-comparison of the predictive ability of each formula because of the lack of a standardised and universally accepted validation procedure. The procedure described in this work can be generalised and extended to the validation of many other nuclear decay formulae or theoretical results. Given the importance of the predictions of the decay half-lives of SHE in the design and execution of the SHE synthesis and radiochemical characterization experiments, it is hoped that the nuclear data community will establish protocols for the analysis of the predictive ability of the different theoretical formulations.

Acknowledgement: The author expresses her gratitude to Prof. Gottfried Münzenberg and Prof. Sigurd Hoffmann of the GSI Helmholtzzentrum für Schwerionenforschung, Darmstadt, Germany, for helpful insights during the $6^{\text {th }}$ International Conference of New Frontiers in Physics, Crete, 2017 and her indebtedness to Prof. Sudip K. Ghosh of the Saha Institute of Nuclear Physics, Kolkata, India, for his illuminating discussions. 


\section{References}

[1] Y. Oganessian. J. Phys.: Conf. Ser. 337012005 (2012)

[2] S. Hoffmann. Eighty years of Research on Super-heavy Nuclei. New Horizons in Fundamental Physics, S. Schramm, M. Schäffer (Eds.). Springer International Publishing, Switzerland (2017).

[3] M. Schädel. Phil. Trans. R. Soc. A373 20140191 (2015).

[4] N. Dasgupta-Schubert, M.A. Reyes, V.A. Tamez. Eur. Phys. J. A, 42, 121 (2009)

[5] G. Royer, J. Phys. G: Nucl. Part. Phys. 26, 1149 (2000)

[6] V.E. Viola jr., G.T. Seaborg, J. Inorg. Nucl. Chem. 28, 741 (1966)

[7] A. Sobiczewski, Z. Patyk, S. Cwiok, Phys. Lett. B 224,1 (1989).

[8] A. Parkhomenko, A. Sobiczewski, Acta Phys. Pol. B 36, 3095 (2005)

[9] Y. Oganessian. (a) J. Phys. G: Nucl. Part. Phys., 34, R165 (2007); (b) "Heavy element research at FLNR (Dubna)", in the $5^{\text {th }}$ International Conference on Exotic Nuclei and

Atomic Masses (ENAM08), Ryn, Poland, September 7-14, 2008.

[10] A. Türler, "Chemistry of superheavy elements", in the $5^{\text {th }}$ International Conference on Exotic Nuclei and Atomic Masses (ENAM08), Ryn, Poland, September 7-14, 2008.

[11] N. Dasgupta-Schubert, M.A. Reyes. At. Data Nucl. Data Tables 93, 907 (2007).

[12] M. Thompson, S.L.R. Ellison, R. Woods. Pure \& Appl. Chem., 74 (5), 835 (2002)

[13] D.A. Skoog, J.J. Leary, Principles of Instrumental Analysis, 4th edition (Saunders College Publishing, Orlando, USA, 1992).

[14] MATHEMATICA 5.2, Wolfram Research Inc., Champaign, Il 61820-7237, USA; W.H. Press, S.A. Teukolsky, W.T. Vetterling, B.P. Flannery, Numerical Recipes in C, Ch. 15-5: Non-linear Models (Cambridge University Press, New York, USA, 2007).

[15] P. Möller, J.R. Nix, W.D. Myers, W.J. Swiatecki, At. Data Nucl. Data Tables, 59 (1995) 185

[16] R-D. Herzberg. Nuclear Structure of Superheavy Elements, in The Chemistry of Superheavy Elements, M. Schädel and D. Shaughnessy (eds.), (Springer Verlag, Berlin, 2014), pp. 83-133.

[17] P. Möller, A.J. Sierk, T. Ichikawa, H. Sagawa, At. Data Nucl. Data Tables, 109-110, 1 (2016)

[18] H. Geiger, J.M. Nuttall, Philos. Mag., 22, 613 (1911) 